\title{
Study Agent Unique Ingredient Identifier
}

National Cancer Institute

\section{Source}

National Cancer Institute. Study Agent Unique Ingredient Identifier. NCI Thesaurus. Code C92646.

The unique ingredient identifier of the investigational product under study. 